\title{
Fuzzy-Logic Based Automatic Control of Hemodynamics
}

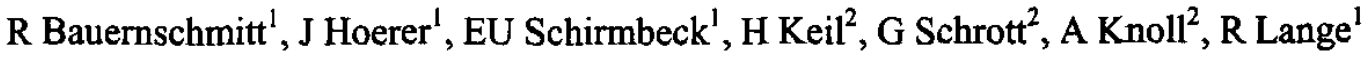 \\ German Heart Center ${ }^{1}$ and Institute for Robotics and Embedded Systems ${ }^{2}$, \\ Technical University of Munich, Germany
}

\begin{abstract}
We propose an automatic closed-loop-system for hemodynamic control on the intensive care unit in patients after cardiac surgery. All possible combinations of four standard hemodynamic parameters (mean arterial pressure, on-line cardiac output, central venous pressure and systemic vascular resistance) and four treatment regimens (fluid administration, noradrenaline, dopmanine and nitrates) were linked by knowledge-based rules in a fuzzy-logic system. After defuzzification, the loop was closed by integration of automatic control of infusion and syringe pumps. The system proved to be able to observe and handle any cardiocirculatory disturbances in circulatory models and animal experiments. The system remained stable throughout any observation period. Hemodynamic changes and therapeutic interventions were documented in an electronic experimental record. In several attempts for automatic therapy control in the past, failures were mainly due to insufficient computer capacity, inadequate sensors and the lack of appropriate mathematical models. We anticipate an improvement of postoperative patient care by induction of therapies and paperless on-line documentation permitting to spend more time to treat the critically ill.
\end{abstract}

\section{Introduction}

Hemodynamic instability after cardiac surgery is a common phenomenon, which usually is managed by timely administration of fluids and inotropic or vasoactive agents. However, due to the growing mass of critically ill patients and the amount of information to be processed, anticipating therapy of the „standard"-patients sometimes is not possible for the intensivist. With the aid of rapid advances in computer technology, controllers for difficult nonlinear problems like the hemodynamic situation of patients immediately after cardiac surgery treated on an intensive care unit (ICU) have been realised [1]. While previous controllers usually were designed as single-input-single-output systems (regulating one hemodynamic parameter with the infusion of one drug [2]), we intended to construct a multiple-input-multipleoutput fuzzy-logic controller, which is able to match the process of clinical decision making very closely and gives the possibility to include additional variables and rules. The evaluation of the controller was performed in an animal experiment, where typical hemodynamic alterations after cardiac surgery were simulated.

\section{Methods}

\subsection{System components}

Hemodynamic data were recorded online by commercially available sensors, A/D-converted, stored and analysed by laptop, which will soon be substituted by a reliable fail-safe embedded computer. The system automatically activates syringe and infusion pumps for application of drugs and volume replacement therapy, if necessary.

\subsection{Expert system and fuzzy controller}

The hemodynamic variables as mean arterial pressure (MAP), central venous pressure (CVP), cardiac output (CO) is easily measurable; in addition, systemic vascular resistance (SVR) is calculated from these data. Based on these data, the application of drugs and volume replacement is controlled by an expert system designed with support of intensive care physicians: Dopamine for positive inotropic effects, noradrenaline for peripheral vasoconstriction and minor positive inotropy, nitrates (Isosorbitdinitrat, ISD) for peripheral vasodilatation and administration of hydroxyethyl starch (HES) for intravascular volume replacement. 
The input variables were classified into three linguistic levels for each variable - low, medium and high -; medium means the input variable is within the normal physiological range. The linguistic terms for each hemodynamic variable can be connected by "and", "or" or "not" and lead to a decision concerning the application of any of the drugs given. Infusion rates are termed "nothing", "little" and "high"; after defuzzification the adequate infusion rate is determined as continuous output by the system. Fuzzy logic offers the advantage of continuous control based on expert knowledge.

Example (for dopamine therapy):

If MAP is normal or high, and CO is normal, and CVP is normal or low, then dopamine: Nothing.

\subsection{Animal experiment}

The first evaluation was performed using digitized flow and pressure curves from prior animal experiments as input signal. The system suggested treatment strategies, serving as a basis for fine-tuning.

The animal experiment was carried out in an anesthetized pig with a body weight of $35 \mathrm{~kg}$. Tip manometers were placed in the descending aorta and the right atrium, cardiac output was measured by a Dopplerflow probe fixed around the pulmonary artery. During the experiment, typical situations occurring during the postoperative course of a patient after open heart surgery were simulated (see results section).

The animal received human care throughout the whole experiment.

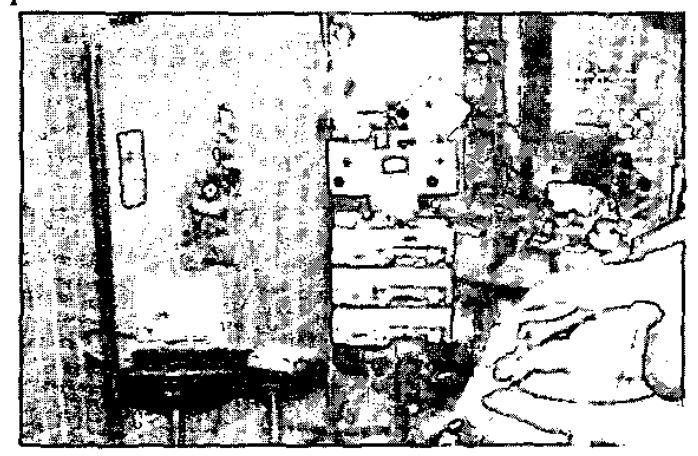

Figure 1. Experimental set-up.

\section{Results}

The objective for the controller is to detect any alterations in hemodynamic parameters and - according to the rules given by the expert system - to counteract these alterations by adequate infusion of drugs or volume replacement. For evaluation of the controller, a set of defined interventions leading to disturbances of stable hemodynamic was performed during the animal experiment. Exemplary original graphs of MAP- and COdata and noradrenaline treatment throughout the whole experiment are given in Figures $2 \mathrm{a}, \mathrm{b}$ and 3.

Steady state (Figures 2 and 3. Time 11.00 - 14.00)

All the measured parameters were within the normal range. The system administered a trace of HES to compensate for small fluid losses caused by mechanical ventilation and urinary output. Noradrenaline was given immediately after induction of anaesthesia (time 11.20) to compensate vasodilatation. Short term peaks of noradrenaline (time 13.00 and 14.00) were due to artefacts in the flow and pressure curves.

Hypertension (Figures 2 and 3. Time 14.50 and 15.00, Figure 4)

Hypertensive periods frequently occur during the postoperative course for a variety of reasons, including peripheral vasoconstriction and/or increased cardiac output. For simulation of a hypertensive crisis, external noradrenaline $\left(1 \mu \mathrm{g} \mathrm{kg}^{-1} \mathrm{~min}^{-1}\right)$ was administered off-loop by continuous infusion, causing a marked elevation of CVP, MAP and SVR. Even CI was increased, which is explained by a partial stimulating effect of noradrenaline on $\beta$-receptors. The system acted by a sharp increase of ISD administration leading to a decrease of CVP, MAP and SVR to normal values within ten minutes. Withdrawal of the noradrenaline infusion after 20 minutes caused a sudden drop of mean arterial pressure and was counteracted by a rapid stop of nitrate and infusion of dopamine and noradrenaline (time 15.10).

Vasodilation (Figures 2 and 3. Time 15.40)

Decreases of peripheral resistance are usually due to the effects of rewarming and the generation of vasoactive cytokines. Experimental vasodilatation was induced by three repetitive injections of a $2 \mathrm{mg}$-bolus of nitrate within two minutes, leading to a drop in MAP, SVR and CVP without significant alteration of $\mathrm{CI}$. The system counteracted by low-dose volume substitution and administration of noradrenaline.

Hemorrhage (Figures 2 and 3. Time 16.10)

In the early postoperative course, when the coagulation system may still be disturbed, patients sometimes suffer from sudden losses of significant amounts of blood. To simulate this condition, $720 \mathrm{ml}$ of blood $(20 \%$ of the animal's estimated total blood volume) was drawn within 8 minutes, resulting in a decrease of all hemodynamic parameters measured. Normal hemodynamic parameters were restored by an initial dose of noradrenaline, a small and short-term application of dopamine and continuous substitution of HES to balance intravascular volume.

Low output (Figures 2 and 3. Time 17.00)

Low cardiac output situations may frequently occur during the early postoperative period due to myocardial reperfusion injury, insufficient coronary perfusion or other reasons. This condition was simulated by injection of a $\beta$-blocker (metoprolol, 15mg) and thiopental (10 
$\mathrm{mg} / \mathrm{kg} / \mathrm{min}$ ), leading to a rise in CVP and a decrease of the remaining parameters. The system administered dopamine and small dose of noradrenalin to stabilize arterial pressure. Nitrate and volume infusion were stopped in the acute phase and restarted after successful management of the initial critical situation.

Figures $2 \mathrm{a}$ and $\mathrm{b}$. Hemodynamic measurements throughout the experiment; $\mathrm{CO}$ and MAP are displayed. For details see text.

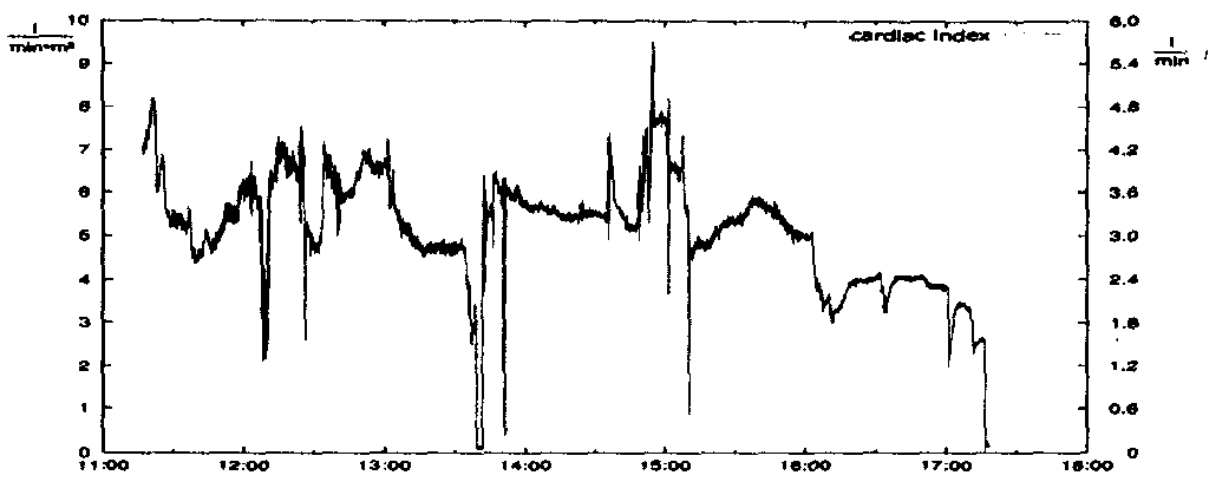

Figure 2a. Cardiac output. See time scale for correlation with the simulation experiments. Drops before time 14.00 are due to artefacts.

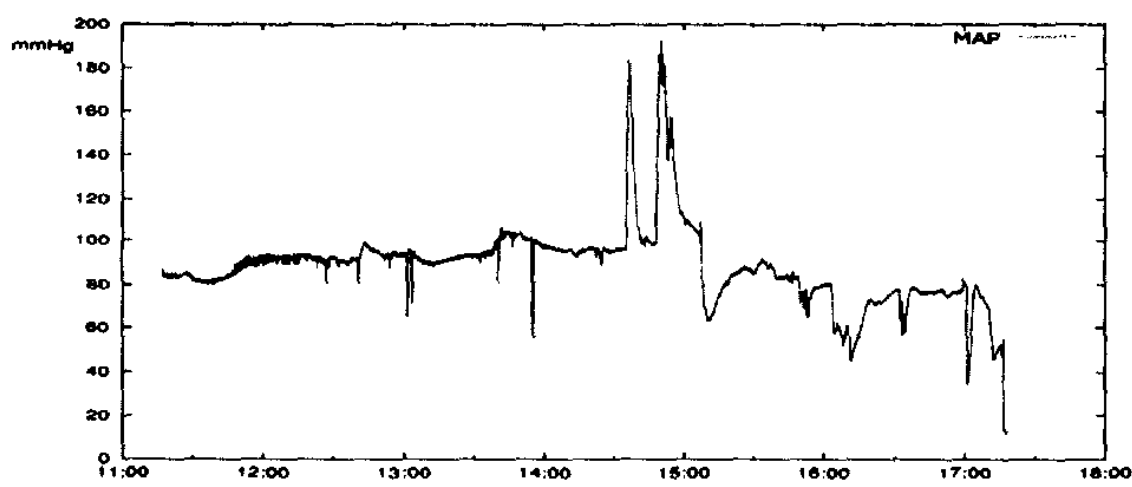

Figure $2 \mathrm{~b}$. Mean arterial pressure. Drops before time 14.00 are due to artefacts.

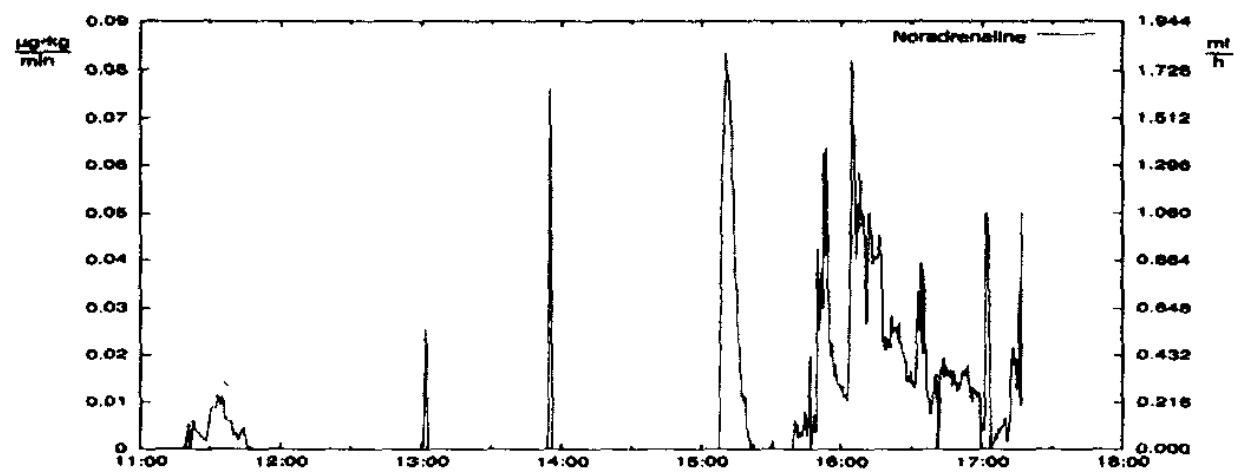

Figure 3. Automatic management of noradrenaline throughout the experiment. Other therapeutic interventions are described in the text. 


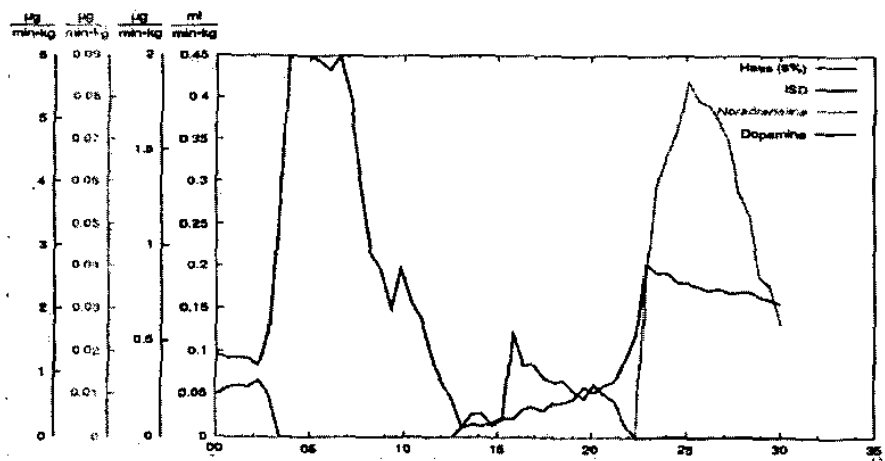

Figure 4. Automatic treatment in case of hypertension. Upper black line: Isosorbiddinitrate (ISD), lower black line: hydroxyethyl starch (HES), grey line: noradrenaline. Hypertension was initially treated by ISD-application (minute 3 to 12). When the external noradrenaline infusion (simulated hypertension) was stopped, the drop of arterial pressure was counteracted by automatic infusion of noradrenaline within the loop ( 22 to 30 minutes).

\section{Discussion}

Postoperative intensive care therapy after cardiac surgery is characterized by standardized regimens in the majority of patients. Even in an uncomplicated patient, there are, however, periods of hemodynamic instability caused by volume shifts from the intravascular to the extravascular space, myocardial reperfusion injuries, generation of vasoactive mediators or hypertension caused by pain and inadequate awakening reactions. Usually these problems can be easily treated, if they are recognized in time, but quite often the attention of the intensive care physician is required by unstable patients with the need for continuous medical support.

The system presented in this paper is designed to support intensive care in the "routine" patient by anticipating and counteracting alterations of the hemodynamic situation before circulatory instability occurs. It is, at least in the present state, not intended to improve the quality of medical decision making, but to remove workload from physicians and nurses and give them more time to treat the critically ill.

Using fuzzy-logic rules based on expert knowledge, this system offers the possibility of continuous control of main hemodynamic parameters. The rules were fixed matching treatment regimens in our ICU, but changes due to special patient conditions or different regimens can easily be realized. Normal alarm functions of the routine monitoring devices remain unaffected and may be set according to the physician's choice.

Our system proved the ability to maintain stable hemodynamics in an animal experiment, although extreme alterations of the circulatory situation were simulated. The four-input - four-output configuration is close to the daily ICU-routine and may facilitate the acceptance among intensive care physicians as compared to a variety of other models.

In this early stage of the development, there are several drawbacks to be overcome. For clinical use, the laptop has to be replaced by a fail-safe, embedded computer network to guarantee maximum safety for the patient.

As can be seen in the original registrations (time 11.00 . 14.00 ), several artefacts in the flow and pressure registrations caused activation of the system. Artefacts occur quite frequently on any ICU due to manipulations at the patient or failure of sensors. Although the duration of drug application was too short to have hemodynamic consequences in the experiment, this activation has to be avoided in the clinical set-up; therefore the integration of adequate artefact detection algorithms is necessary.

Including more input and output variables, which no doubt is desirable, would lead to a huge increase in the number of rules. Therefore our present work is directed towards the development of a self-learning system taking into consideration important parameters like depth of narcosis and mechanical ventilation.

\section{References}

[1] Huang JW, Roy RJ: Multiple-drug hemodynamic control using fuzzy decision theory. IEEE Trans Biomed Eng 1998, 45 (2): 213-228

[2] Ying H, Sheppard LC: Real-time expert-system-based fuzzy control of mean arterial pressure in pigs with sodium nitroprusside infusion. Med Prog Through Technol 1990, 16: $69-76$

Address for correspondence.

PD Dr. med. R. Bauernschmitt.

German Heart Center Munich.

Clinic for Cardiovascular Surgery.

Lazarettstr. 36, 80636 Munich, Germany.

bauernschmitt@dhm.mhn.de. 